\title{
Structure-Guided Design of Cryptococcus neoformans Protein Farnesyltransferase Inhibitors with Antifungal Activity You Wang ${ }^{1}$, Lorena Beese ${ }^{2}$ \\ ${ }^{1}$ Duke University ${ }^{2}$ Duke University Medical Center \\ you.wang@duke.edu
}

Invasive fungal infections are pervasive and often life threatening, resulting in over 11.5 million life-threatening infections. Cryptococcus neoformans is a major human fungal pathogen causing over 160,000 deaths each year. The options for threating C. neoformans infections are limited and the drug resistance is emerging and spreading. Therefore, there is a need for new therapeutics. Protein farnesyltransferase (FTase) is essential for the virulence of C. neoformans, providing opportunities to develop species specific FTase inhibitors for treating infectious diseases.

Here we report the development of potent antifungal compounds using a previous human FTase inhibitor as the chemical scaffold for derivatization. While this compound is stable and bioavailable in animal models, it failed to show significant fungal growth inhibition. Successive rounds of structure guided optimization led to a novel compound that shows potent inhibition of $\mathrm{C}$. neoformans grown with MIC of $3 \mu \mathrm{M}$. The IC50 of the compound is $0.7 \mu \mathrm{M}$, and decreases to approximately $0.1 \mu \mathrm{M}$ in the presence of physiological concentration of phosphate. Crystal structures of the compound bound to CnFTase show a distinct binding mode from the starting compound, illustrating a bisubstrate inhibition mechanism. The compound also shows a RAS mis-localization pattern that is similar to inactive mutant protein further validating the mechanism of inhibition. Additionally, the compound does not exhibit significant toxicity up to $200 \mu \mathrm{M}$ in mammalian cell based assays.

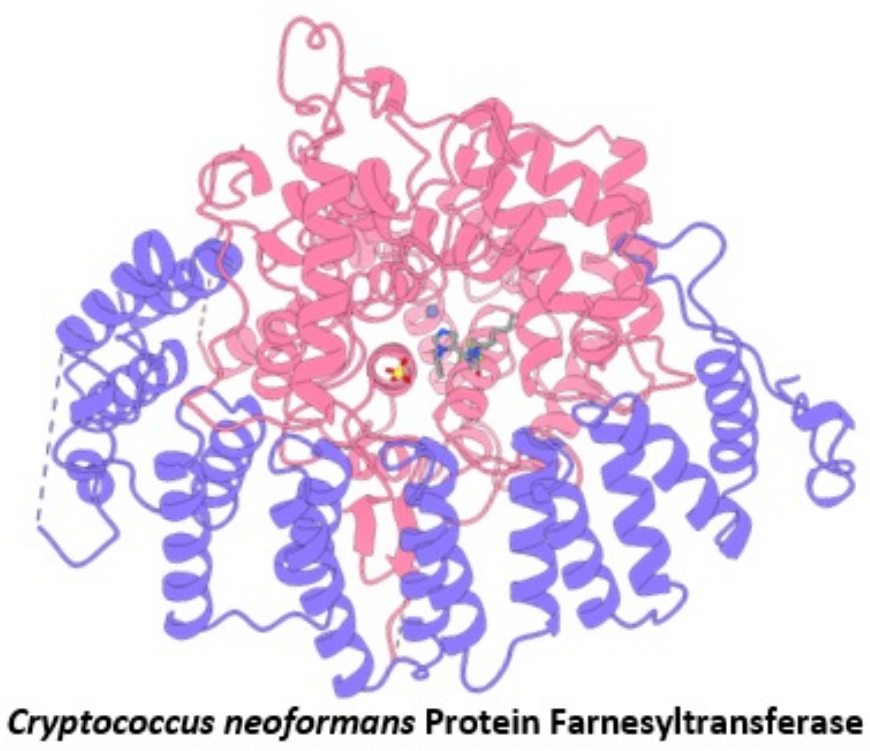

Figure 1 